University of Nebraska - Lincoln

DigitalCommons@University of Nebraska - Lincoln

Faculty Publications in Computer \& Electronics Electrical \& Computer Engineering, Department Engineering (to 2015)

2008

\title{
The Theory of Quaternion Orthogonal Designs
}

\author{
Jennifer Seberry \\ University of Wollongong \\ Ken Finlayson \\ University of Wollongong \\ Sarah Spence Adams \\ Franklin W. Olin College of Engineering \\ Tadeusz Wysocki \\ University of Nebraska-Lincoln, wysocki@uow.edu.au \\ Tianbing Xia \\ University of Wollongong \\ See next page for additional authors
}

Follow this and additional works at: https://digitalcommons.unl.edu/computerelectronicfacpub

Part of the Computer Engineering Commons

Seberry, Jennifer; Finlayson, Ken; Adams, Sarah Spence; Wysocki, Tadeusz; Xia, Tianbing; and Wysocki, Beata Joanna, "The Theory of Quaternion Orthogonal Designs" (2008). Faculty Publications in Computer \& Electronics Engineering (to 2015). 13.

https://digitalcommons.unl.edu/computerelectronicfacpub/13

This Article is brought to you for free and open access by the Electrical \& Computer Engineering, Department of at DigitalCommons@University of Nebraska - Lincoln. It has been accepted for inclusion in Faculty Publications in Computer \& Electronics Engineering (to 2015) by an authorized administrator of DigitalCommons@University of Nebraska - Lincoln. 


\section{Authors}

Jennifer Seberry, Ken Finlayson, Sarah Spence Adams, Tadeusz Wysocki, Tianbing Xia, and Beata Joanna Wysocki 


\title{
The Theory of Quaternion Orthogonal Designs
}

\author{
Jennifer Seberry, Senior Member, IEEE, Ken Finlayson, Sarah Spence Adams, Member, IEEE, \\ Tadeusz Antoni Wysocki, Senior Member, IEEE, Tianbing Xia, and Beata Joanna Wysocki
}

\begin{abstract}
Over the past several years, there has been a renewed interest in complex orthogonal designs for their application in space-time block coding. Motivated by the success of this application, this paper generalizes the definition of complex orthogonal designs by introducing orthogonal designs over the quaternion domain. This paper builds a theory of these novel quaternion orthogonal designs, offers examples, and provides several construction techniques. These theoretical results, along with the results of preliminary simulations, lay the foundation for developing applications of these designs as orthogonal space-time-polarization block codes.
\end{abstract}

Index Terms-Diversity methods, mobile communications, multiple-input multiple-output (MIMO) systems, polarization.

\section{INTRODUCTION: Motivation AND PRELIMINARIES}

$\mathbf{S}^{\mathrm{p}}$ PACE-TIME block codes built from generalized complex orthogonal designs can be viewed as a generalization of Alamouti's scheme [1] and were introduced by Tarokh et al. [18]. These space-time block codes are particularly attractive because they can provide full transmit diversity while requiring a very simple decoupled maximum-likelihood (ML) decoding method [13], [18]. The combination of space and time diversity moved the capacity of wireless communication systems towards the theoretical limits, and this technique has been adopted in the 3G standard in the form of an Alamouti code and in the newly proposed standard for wireless LANs IEEE $802.11 \mathrm{n}$. The success of the combined diversity achieved by complex orthogonal space-time block codes motivated us to consider alternative definitions of orthogonal designs that would permit the combination of space and time diversity with additional forms of diversity. We expect that the combination of additional forms of diversity, for example, frequency and/or polarization diversity, will further improve capacity.

Manuscript received April 20, 2006; revised June 16, 2007. The associate editor coordinating the review of this manuscript and approving it for publication was Dr. Michael Buehrer. This work was supported in part by NSA Grant H98230-07-1-0022 and an NSF-AWM Mentoring Travel Grant.

J. Seberry, K. Finlayson, and T. Xia are with the Centre for Computer and Information Security Research, School of Computer Science and Software Engineering, University of Wollongong, NSW 2522, Australia (e-mail: jennie@uow. edu.au; kaf03@uow.edu.au; txia@uow.edu.au).

S. S. Adams is with the Franklin W. Olin College of Engineering, Olin Way, Needham, MA 02492-1245 USA (e-mail: sarah.adams@olin.edu).

T. A. Wysocki was with the School of Electrical, Computer and Telecommunications Engineering, University of Wollongong, NSW 2522, Australia. He is now with the Peter Kiewit Insitute, University of Nebraska—Lincoln, Omaha, NE 68588 USA (e-mail: twysocki@mail.unomaha.edu).

B. J. Wysocki is with the School of Electrical, Computer and Telecommunications Engineering, University of Wollongong, NSW 2522, Australia (e-mail: bjw@uow.edu.au).

Color versions of one or more of the figures in this paper are available online at http://ieeexplore.ieee.org.

Digital Object Identifier 10.1109/TSP.2007.906773
Polarization diversity has been studied in the past [4], and Collins has provided a detailed assessment of the diversity gain under Rayleigh fading [5]. Recently, the demand for high rates in mobile communications has raised interest in applying polarization diversity, often together with other forms of diversity. It has been shown that polarization diversity can significantly add to the performance improvements offered by other diversity techniques and be nearly as effective as spatial diversity for base station antennas without a noticeable increase in their dimensions [6], [14]. In addition, the use of dual-polarized antennas for both transmitter and receiver combined with channel coding has been previously studied [22]. Polarization diversity is usually considered separately from the other forms of diversity and there is no well known mechanism of utilizing it jointly with the other forms rather than through a simple concatenation. However, it is intuitively clear, and shown through space-time coding, that it is possible to achieve better performance by using combined rather than concatenated forms of diversity.

The work of Isaeva and Sarytchev [12] implies that a signal having components in two orthogonal polarizations can be modeled by means of quaternions, whereby two complex constellations orthogonal to each other on a polarization plane form a quaternion, or hypercomplex, number [2]. Using this notation, any rotation on the polarization plane can be represented in a similar way as a rotation on a complex plane. Similarly, any change to the polarization bases, e.g., due to differences between transmit and receive antenna alignments, can be represented in the quaternion notation as long as the polarization bases are orthogonal [12].

Hence, we propose to develop orthogonal designs with quaternion elements to be used as a basis for orthogonal space-time-polarization block codes. The generalization from real orthogonal designs to complex orthogonal designs was straight-forward, while this further generalization is significantly complicated by the non-commuting nature of the quaternions. The proposed application of these quaternion designs would allow polarization diversity to be jointly combined with space and time diversities. Our preliminary simulations indicate that the proposed quaternion orthogonal space-time-polarization block codes perform similarly to complex orthogonal space-time block codes that use twice as many transmit antennas and twice as many receive antennas. In addition to further studying this savings on dimension, other potential performance gains must be further addressed in future work.

The original definition of orthogonal design proposed by Geramita, Geramita, and Seberry Wallis [10] concerned only square matrices defined on real variables. An orthogonal design (OD) of order $n$ and type $\left(s_{1}, s_{2}, \ldots, s_{u}\right)$ on commuting real variables $x_{1}, x_{2}, \ldots, x_{u}$, denoted $\mathrm{OD}\left(n ; s_{1}, s_{2}, \ldots, s_{u}\right)$, is an 
$n \times n$ matrix $A$ with entries in the set $\left\{0, \pm x_{1}, \pm x_{2}, \ldots, \pm x_{u}\right\}$ satisfying $A A^{T}=\left(\sum_{h=1}^{u} s_{h} x_{h}^{2}\right) I_{n}$, where $T$ denotes the transpose of a matrix and $I_{n}$ is the identity matrix of order $n$. Qualitatively, we say that the rows of $A$ are formally orthogonal and each row has precisely $s_{h}$ entries of the type $\pm x_{h}$. Geramita, Geramita and Seberry Wallis also showed that $A^{T} A=\left(\sum_{h=1}^{u} s_{h} x_{h}^{2}\right) I_{n}$, and so our qualitative description of $A$ applies equally well to the columns of $A$ [10]. Geramita and Geramita [11] first studied orthogonal designs in the complex domain as $n \times n$ matrices $A$ of type $\left(s_{1}, s_{2}, \ldots, s_{u}\right)$ with entries in the set $\left\{0, \pm x_{1}, \pm \mathbf{i} x_{1}, \pm x_{2}, \pm \mathbf{i} x_{2}, \ldots, \pm x_{u}, \pm \mathbf{i} x_{u}\right\}$ satisfying $A^{H} A=A A^{H}=\left(\sum_{h=1}^{u} s_{h} x_{h}^{2}\right) I_{n}$, where $H$ denotes the Hermitian transpose. This definition can be generalized to include $r \times n$ rectangular designs. We say that these designs are complex orthogonal designs (CODs) on real variables $x_{1}, x_{2}, \ldots, x_{u}$. A variation more widely used in the signals processing literature is the generalized complex orthogonal design $A$ of size $r \times n$ and type $\left(s_{1}, s_{2}, \ldots, s_{u}\right)$ with entries in the set of complex variables $\left\{0, \pm z_{1}, \pm z_{1}^{*}, \pm \mathbf{i} z_{1}, \pm \mathbf{i} z_{1}^{*}, \pm z_{2}, \pm z_{2}^{*}, \pm \mathbf{i} z_{2}, \pm \mathbf{i} z_{2}^{*}, \ldots, \pm z_{u}\right.$, $\left.\pm z_{u}^{*}, \pm \mathbf{i} z_{u}, \pm \mathbf{i} z_{u}^{*}\right\}$ satisfying $A^{H} A=\left(\sum_{h=1}^{u} s_{h}\left|z_{h}\right|^{2}\right) I_{n}$ [13], [18]. We say that these designs are CODs on complex variables $z_{1}, z_{2}, \ldots, z_{u}$. A number of variations on these definitions has been considered. For example, if an entry of a design is a linear combination of the permitted variables, then the design is said to be with linear processing.

We recall here the properties of the quaternions that are critical to our discussion and refer the reader to Altmann's book [2] for more information. The noncommutative quaternions $\mathcal{Q}=$ $\{ \pm 1, \pm \mathbf{i}, \pm \mathbf{j}, \pm \mathbf{k}\}$ satisfy $\mathbf{i}^{2}=\mathbf{j}^{2}=\mathbf{k}^{2}=\mathbf{i j k}=-1$. A quaternion variable $\mathbf{a}=a_{1}+a_{2} \mathbf{i}+a_{3} \mathbf{j}+a_{4} \mathbf{k}$, where $a_{1}, a_{2}$, $a_{3}, a_{4}$ are real variables has a quaternion conjugate defined by $\mathbf{a}^{Q}=a_{1}-a_{2} \mathbf{i}-a_{3} \mathbf{j}-a_{4} \mathbf{k}$. It follows that $\mathbf{a}^{Q} \mathbf{a}=\mathbf{a a}^{Q}=|\mathbf{a}|^{2}$ is real. Given a matrix $A=\left(\mathbf{a}_{\ell, m}\right)$, where $\mathbf{a}_{\ell, m}$ are quaternion variables, its quaternion transpose is $A^{Q}=\left(\mathbf{a}_{m, \ell}^{Q}\right)$. It follows from this definition of the quaternion transpose that the product $A^{Q} B$ consists of taking the inner product of the columns of $A$ and the columns of $B$, where the inner product of quaternion variables $\mathbf{a}$ and $\mathbf{b}$, is said to be $\mathbf{a} \cdot \mathbf{b}=\mathbf{a}^{Q} \mathbf{b}$. This notion will now be developed as we define quaternion matrices whose columns are mutually orthogonal under this inner product.

Definition 1: A quaternion orthogonal design (QOD) on commuting real variables $x_{1}, x_{2}, \ldots, x_{u}$ of type $\left(s_{1}, s_{2}, \ldots, s_{u}\right)$ is an $r \times n$ matrix $A$ with entries from $\left\{0, \pm \mathbf{q}_{1} x_{1}, \pm \mathbf{q}_{2} x_{2}, \ldots, \pm \mathbf{q}_{u} x_{u}\right\}, \mathbf{q}_{h} \in \mathcal{Q}$, that satisfies $A^{Q} A=\left(\sum_{h=1}^{u} s_{h} x_{h}^{2}\right) I_{n}$. This design is denoted by $\operatorname{QOD}\left(r, n ; s_{1}, s_{2}, \ldots, s_{u}\right)$. When $r=n$, we have $A^{Q} A=A A^{Q}=\left(\sum_{h=1}^{u} s_{h} x_{h}^{2}\right) I_{n}$. Similarly, we define a QOD $A$ on commuting complex variables $z_{1}, z_{2}, \ldots, z_{u}$ to have entries from the set $\left\{0, \pm z_{1}, \pm z_{1}^{*}, \pm z_{2}, \pm z_{2}^{*}, \ldots, \pm z_{u}, \pm z_{u}^{*}\right\}$ including possible multiplications on the left and/or right by quaternion elements $\mathbf{q} \in \mathcal{Q}$, and to satisfy $A^{Q} A=$ $\left(\sum_{h=1}^{u} s_{h}\left|z_{h}\right|^{2}\right) I_{n}$. Finally, we define a QOD $A$ on noncommuting quaternion variables $\mathbf{a}_{1}, \mathbf{a}_{2}, \ldots, \mathbf{a}_{u}$ to have entries from $\left\{0, \pm \mathbf{a}_{1}, \pm \mathbf{a}_{1}^{Q}, \pm \mathbf{a}_{2}, \pm \mathbf{a}_{2}^{Q}, \ldots, \pm \mathbf{a}_{u}, \pm \mathbf{a}_{u}^{Q}\right\}$ including possible multiplications on the left and/or right by quaternion elements $\mathbf{q} \in \mathcal{Q}$ and to satisfy $A^{Q} A=\left(\sum_{h=1}^{u} s_{h}\left|\mathbf{a}_{h}\right|^{2}\right) I_{n}$. We can generalize these definitions to allow the design entries to be real linear combinations of the permitted variables and their quaternion multipliers, in which case we say the design is with linear processing.

$$
\text { Example 2: The matrix } X=\left[\begin{array}{cc}
-x_{1} & x_{2} \mathbf{i} \\
-x_{2} \mathbf{j} & x_{1} \mathbf{k}
\end{array}\right] \text { is a QOD on }
$$
real variables $x_{1}, x_{2}$. The matrix $Z=\left[\begin{array}{cc}\mathbf{i} z_{1} & \mathbf{i} z_{2} \\ -\mathbf{j} z_{2}^{*} & \mathbf{j} z_{1}^{*}\end{array}\right]$ is a QOD on complex variables $z_{1}, z_{2}$. Finally $A=\left[\begin{array}{cc}\mathbf{a} & 0 \\ 0 & \mathbf{a}\end{array}\right]$ is the most obvious example of a QOD on the quaternion variable $\mathrm{a}$. QODs on quaternion variables are the most difficult to construct.

In Section II, we provide two construction techniques that utilize real and complex orthogonal designs to build quaternion orthogonal designs. In Section III, we define quaternion-commutivity and quaternion amicable designs in an effort to develop construction techniques that do not rely on existing real and complex orthogonal designs. In Section IV, we describe simulation results of a quaternion orthogonal design applied as a space-time-polarization block code. The paper is concluded and some open problems are described in Section V.

\section{Construction Techniques Using REAL AND COMPLEX DESIGNS}

In this section, we provide two construction techniques that use existing real and complex orthogonal designs to build quaternion orthogonal designs. The constructions demonstrate that QODs exist, and the resulting examples help build intuition concerning the behavior of these novel designs. The noncommutativity of the quaternions prevents simple generalizations of construction techniques for ODs or CODs. However, the new techniques that we propose for constructing QODs can be readily modified to construct ODs or CODs.

In Section II-A, we define quaternion permutation matrices and use these matrices, along with existing ODs and CODs, to construct QODs. In Section II-B, we define symmetric-paired complex orthogonal designs and use these designs to build QODs.

\section{A. Quaternion Permutation Matrices}

We present here our most simple, and potentially most useful, method for constructing quaternion orthogonal designs. The construction requires only readily available real or complex orthogonal designs and what we define below as quaternion permutation matrices.

Definition 3: An $n \times n$ matrix $M$ is a quaternion permutation matrix if it has one nonzero element $\mathbf{q} \in \mathcal{Q}$ per row and per column and if $M^{Q} M=M M^{Q}=I_{n}$.

Theorem 4: Let $D$ be an $r \times n$ orthogonal design or complex orthogonal design of type $\left(s_{1}, s_{2}, \ldots, s_{u}\right)$. Let $M$ be an $r \times r$ and $N$ be an $n \times n$ quaternion permutation matrix. Then $M D N$ is an $r \times n$ quaternion orthogonal design of type $\left(s_{1}, s_{2}, \ldots, s_{u}\right)$.

Proof: If $D$ is real, write $D^{Q} D=D^{T} D=$ $\sum_{h=1}^{u} s_{h}\left|x_{h}\right|^{2} I_{n}=\gamma I_{n}$. If $D$ is complex, write 
$D^{Q} D=D^{H} D=\sum_{h=1}^{u} s_{h}\left|z_{h}\right|^{2} I_{n}=\gamma I_{n}$. In either case, the coefficient $\gamma$ is real. Now, consider

$$
\begin{aligned}
(M D N)^{Q}(M D N) & =\left(N^{Q} D^{Q} M^{Q}\right)(M D N) \\
& =N^{Q} D^{Q} M^{Q} M D N \\
& =N^{Q} \gamma I_{n} N=\gamma N^{Q} N \\
& =\gamma I_{n}
\end{aligned}
$$

which clearly satisfies the required orthogonality constraint so that $M D N$ is a quaternion orthogonal design.

We note that Theorem 4 holds for any quaternion matrices $M$ and $N$ such that $M^{Q} M=I_{r}$ and $N^{Q} N=I_{n}$. Our restriction to matrices that have exactly one nonzero quaternion element per column and per row is meant to show the simplicity of this idea and to facilitate the construction of such quaternion permutation matrices. This construction shows that orthogonal designs over the quaternion domain exist and can be constructed in a straightforward way.

Example 5: For simplicity, let $N$ be the identity matrix. Let $M=\left[\begin{array}{cc}\mathbf{i} & 0 \\ 0 & -\mathbf{j}\end{array}\right]$. Let $D=\left[\begin{array}{cc}x_{1} & x_{2} \\ x_{2} & -x_{1}\end{array}\right]$ be an OD on real variables $x_{1}, x_{2}$. Then, $M D=\left[\begin{array}{cc}x_{1} \mathbf{i} & x_{2} \mathbf{i} \\ -x_{2} \mathbf{j} & x_{1} \mathbf{j}\end{array}\right]$ is QOD on real variables $x_{1}, x_{2}$. Now, let $D^{\prime}=\left[\begin{array}{cc}x_{1} \mathbf{i} & x_{2} \\ x_{2} & x_{1} \mathbf{i}\end{array}\right]$ be a COD on real variables $x_{1}, x_{2}$. Then, $M D^{\prime}=\left[\begin{array}{cc}-x_{1} & x_{2} \mathbf{i} \\ -x_{2} \mathbf{j} & x_{1} \mathbf{k}\end{array}\right]$ is a QOD on real variables $x_{1}, x_{2}$. Finally, let $D^{\prime \prime}=\left[\begin{array}{cc}z_{1} & z_{2} \\ z_{2}^{*} & -z_{1}^{*}\end{array}\right]$ be a COD on complex variables $z_{1}, z_{2}$. Then, $M D^{\prime \prime}=\left[\begin{array}{cc}\mathbf{i} z_{1} & \mathbf{i} z_{2} \\ -\mathbf{j} z_{2}^{*} & \mathbf{j} z_{1}^{*}\end{array}\right]$ is a QOD on complex variables $z_{1}, z_{2}$.

\section{B. Symmetric-Paired Designs}

We now present a construction technique that uses specially chosen pairs of complex orthogonal designs of size $r \times n$ to build $r \times n$ quaternion orthogonal designs.

Definition 6: Two complex orthogonal designs $A$ and $B$ are said to be symmetric-paired designs if $A B^{H}$ is symmetric and/or if $A^{H} B$ is symmetric.

We recall that complex amicable designs $A$ and $B$ satisfy $A B^{H}=B A^{H}$ and/or $A^{H} B=B^{H} A$ [21]. Hence, the proposed definition of symmetric-paired designs has a resemblance to amicability. The concept of amicability is revisited in Section III-B.

Lemma 7: Let $A$ and $B$ be matrices whose entries include 0 and arbitrary complex variables (for example, $A$ and $B$ could be complex orthogonal designs). If $A B^{H}$ is a symmetric matrix, then $A B^{H} \mathbf{q}=\mathbf{q} B A^{H}$, where $\mathbf{q} \in\{ \pm \mathbf{j}, \pm \mathbf{k}\}$. Similarly, if $A^{H} B$ is a symmetric matrix, then $A^{H} B \mathbf{q}=\mathbf{q} B^{H} A$, where $\mathbf{q} \in\{ \pm \mathbf{j}, \pm \mathbf{k}\}$.

Proof: Suppose that $A B^{H}$ is a symmetric matrix and denote $A B^{H}=\left[\alpha_{s t}\right]$, where $\alpha_{s t}=a_{s t}+b_{s t} \mathbf{i}$ is a complex variable and so $a_{s t}, b_{s t}$ are real variables. Then $B A^{H}=\left(A B^{H}\right)^{H}=$ $\left(\left[\alpha_{s t}\right]\right)^{H}=\left[\alpha_{t s}^{*}\right]$. Thus

$$
\begin{aligned}
\mathbf{q} B A^{H}-A B^{H} \mathbf{q} & =\mathbf{q}\left[\alpha_{t s}^{*}\right]-\left[\alpha_{s t}\right] \mathbf{q} \\
& =\left[\mathbf{q}\left(a_{t s}-b_{t s} \mathbf{i}\right)\right]-\left[\left(a_{s t}+b_{s t} \mathbf{i}\right) \mathbf{q}\right] \\
& =\left[\mathbf{q}\left(a_{t s}-a_{s t}\right)-\mathbf{q} \mathbf{i}\left(b_{t s}\right)-\mathbf{i} \mathbf{q}\left(b_{s t}\right)\right] \\
& =0
\end{aligned}
$$

which follows since the symmetry of $A B^{H}$ implies that $a_{t s}=$ $a_{s t}$ and $b_{t s}=b_{s t}$, and since $\mathbf{i q}=-\mathbf{q} \mathbf{i}$ for $\mathbf{q} \in\{ \pm \mathbf{j}, \pm \mathbf{k}\}$. It follows that $\mathbf{q} B A^{H}=A B^{H} \mathbf{q}$, where $\mathbf{q} \in\{ \pm \mathbf{j}, \pm \mathbf{k}\}$.

It follows similarly that if $A^{H} B$ is symmetric, then $\mathbf{q} B^{H} A=$ $A^{H} B \mathbf{q}$, where $\mathbf{q} \in\{ \pm \mathbf{j}, \pm \mathbf{k}\}$.

Example 8: Consider the following CODs on real variables $x_{1}, x_{2}, x_{3}, x_{4}: A=\left[\begin{array}{cc}x_{1} & x_{2} \mathbf{i} \\ x_{2} \mathbf{i} & x_{1}\end{array}\right]$ and $B=\left[\begin{array}{cc}x_{3} & x_{4} \mathbf{i} \\ x_{4} \mathbf{i} & x_{3}\end{array}\right]$. One can confirm that

$$
A B^{H}=\left[\begin{array}{cc}
x_{1} x_{3}+x_{2} x_{4} & \left(-x_{1} x_{4}+x_{2} x_{3}\right) \mathbf{i} \\
\left(x_{2} x_{3}-x_{1} x_{4}\right) \mathbf{i} & x_{1} x_{3}+x_{2} x_{4}
\end{array}\right]
$$

is symmetric. Lemma 7 then implies that $A B^{H} \mathbf{j}=\mathbf{j} B A^{H}$, which we now confirm directly:

$$
\begin{aligned}
A B^{H} \mathbf{j} & =\left[\begin{array}{cc}
x_{1} x_{3}+x_{2} x_{4} & \left(-x_{1} x_{4}+x_{2} x_{3}\right) \mathbf{i} \\
\left(x_{2} x_{3}-x_{1} x_{4}\right) \mathbf{i} & x_{1} x_{3}+x_{2} x_{4}
\end{array}\right] \mathbf{j} \\
& =\left[\begin{array}{cc}
\left(x_{1} x_{3}+x_{2} x_{4}\right) \mathbf{j} & \left(-x_{1} x_{4}+x_{2} x_{3}\right) \mathbf{k} \\
\left(x_{2} x_{3}-x_{1} x_{4}\right) \mathbf{k} & \left(x_{1} x_{3}+x_{2} x_{4}\right) \mathbf{j}
\end{array}\right] \\
& =\mathbf{j}\left[\begin{array}{cc}
x_{1} x_{3}+x_{2} x_{4} & \left(x_{1} x_{4}-x_{2} x_{3}\right) \mathbf{i} \\
\left(x_{1} x_{4}-x_{2} x_{3}\right) \mathbf{i} & x_{1} x_{3}+x_{2} x_{4}
\end{array}\right] \\
& =\mathbf{j} B A^{H} .
\end{aligned}
$$

It is similar to show that $A^{H} B$ is also symmetric, which implies that $\mathbf{j} B^{H} A=A^{H} B \mathbf{j}$.

Theorem 9: Let $A$ and $B$ be complex orthogonal designs $\operatorname{COD}\left(n, n ; s_{1}, s_{2}, \ldots, s_{u}\right)$ and $\operatorname{COD}\left(n, n ; t_{1}, t_{2}, \ldots, t_{u}\right)$ respectively, on commuting complex variables $z_{1}, \ldots, z_{u}$. If $A^{H} B$ is symmetric, then $A+B \mathbf{j}$ is a quaternion orthogonal design $\operatorname{QOD}\left(n, n ; s_{1}+t_{1}, s_{2}+t_{2}, \ldots, s_{u}+t_{u}\right)$ on the complex variables $z_{1}, \ldots, z_{u}$. Specifically, $(A+B \mathbf{j})^{Q}(A+B \mathbf{j})=$ $\sum_{h=1}^{u}\left(s_{h}+t_{h}\right)\left|z_{h}\right|^{2} I_{n}$.

Proof: Since $A$ and $B$ are complex, we have $A^{Q}=A^{H}$ and $B^{Q}=B^{H}$. Then

$$
\begin{aligned}
(A+B \mathbf{j})^{Q}(A+B \mathbf{j}) & =\left(A^{Q}-\mathbf{j} B^{Q}\right)(A+B \mathbf{j}) \\
& =A^{Q} A-\mathbf{j} B^{Q} A+A^{Q} B \mathbf{j}-\mathbf{j} B^{Q} B \mathbf{j} \\
& =A^{Q} A+B^{Q} B-\mathbf{j} B^{Q} A+A^{Q} B \mathbf{j} \\
& =A^{H} A+B^{H} B-\mathbf{j} B^{H} A+A^{H} B \mathbf{j} \\
& =A^{H} A+B^{H} B \text { (by Lemma 7) } \\
& =\sum_{h=1}^{u} s_{h} z_{h} z_{h}^{*} I+\sum_{h=1}^{u} t_{h} z_{h} z_{h}^{*} I \\
& =\sum_{h=1}^{u}\left(s_{h}+t_{h}\right)\left|z_{h}\right|^{2} I .
\end{aligned}
$$


Hence, if $A$ and $B$ are CODs on complex variables $z_{1}, z_{2}, \ldots, z_{u}$ and $A^{H} B$ is symmetric, then $A+B \mathbf{j}$ is a quaternion orthogonal design on complex variables $z_{1}, z_{2}, \ldots, z_{u}$.

Corollary 10: Let $A$ and $B$ be complex orthogonal designs $\operatorname{COD}\left(n, n ; s_{1}, s_{2}, \ldots, s_{u}\right)$ and $\operatorname{COD}\left(n, n ; t_{1}, t_{2}, \ldots, t_{u}\right)$ respectively, on commuting real variables $x_{1}, \ldots, x_{u}$. If $A^{H} B$ is symmetric, then $A+B \mathbf{j}$ is a quaternion orthogonal design $Q O D\left(n, n ; s_{1}+t_{1}, s_{2}+t_{2}, \ldots, s_{u}+t_{u}\right)$ on real variables $x_{1}, \ldots, x_{u}$. Specifically, $(A+B \mathbf{j})^{Q}(A+B \mathbf{j})=$ $\sum_{h=1}^{u}\left(s_{h}+t_{h}\right) x_{h}^{2} I_{n}$.

Example 11: The designs $A=\left[\begin{array}{cc}z_{1} & z_{2} \\ -z_{2}^{*} & z_{1}^{*}\end{array}\right]$ and $B=\left[\begin{array}{cc}z_{2} & z_{1} \\ z_{1}^{*} & -z_{2}^{*}\end{array}\right]$, where $z_{1}$ and $z_{2}$ are commuting complex variables, are $\operatorname{COD}(2,2 ; 1,1)$ designs that are equivalent to Alamouti's code [1]. It is straightforward to verify that $A^{H} B$ is symmetric. Then, $A+B \mathbf{j}$ gives $\left[\begin{array}{cc}z_{1}+z_{2} \mathbf{j} & z_{2}+z_{1} \mathbf{j} \\ -z_{2}^{*}+z_{1}^{*} \mathbf{j} & z_{1}^{*}-z_{2}^{*} \mathbf{j}\end{array}\right]$, which is a $\operatorname{QOD}(2,2 ; 2,2)$ with linear processing on complex variables $z_{1}, z_{2}$. Defining a quaternion variable $\mathbf{a}=z_{1}+z_{2} \mathbf{j}$ shows that $A+B \mathbf{j}=\left[\begin{array}{cc}\mathbf{a} & -\mathbf{i a k} \\ \mathbf{j a} & -\mathbf{k a k}\end{array}\right]$. Hence, $A+B \mathbf{j}$ can also be considered a QOD on the one quaternion variable $\mathbf{a}$.

Example 12: Since our examples have been restricted to $2 \times 2$ designs thus far, we provide an example using maximum rate, minimum decoding delay CODs of size $4 \times 4$. To show variety, we allow a scalar multiple of one of our complex variables.

$$
A=\left[\begin{array}{cccc}
z_{3} & 0 & -z_{1} & -2 z_{2} \\
0 & z_{3} & 2 z_{2}^{*} & -z_{1}^{*} \\
z_{1}^{*} & -2 z_{2} & z_{3}^{*} & 0 \\
2 z_{2}^{*} & z_{1} & 0 & z_{3}^{*}
\end{array}\right]
$$

is a COD satisfying $A^{H} A=\left(\left|z_{1}\right|^{2}+4\left|z_{2}\right|^{2}+\left|z_{3}\right|^{2}\right) I$. Also

$$
B=\left[\begin{array}{cccc}
-z_{1} & -2 z_{2} & z_{3} & 0 \\
2 z_{2}^{*} & -z_{1} & 0 & z_{3} \\
z_{3}^{*} & 0 & z_{1}^{*} & -2 z_{2} \\
0 & z_{3}^{*} & 2 z_{2}^{*} & z_{1}^{*}
\end{array}\right]
$$

is a COD satisfying $B^{H} B=\left(\left|z_{1}\right|^{2}+4\left|z_{2}\right|^{2}+\left|z_{3}\right|^{2}\right) I$. It follows that

$$
\begin{aligned}
A^{H} B & =\left(\left|z_{1}\right|^{2}+4\left|z_{2}\right|^{2}+\left|z_{3}\right|^{2}\right)\left[\begin{array}{cccc}
0 & 0 & 1 & 0 \\
0 & 0 & 0 & 1 \\
1 & 0 & 0 & 0 \\
0 & 1 & 0 & 0
\end{array}\right] \\
& =\left(A^{H} B\right)^{T}
\end{aligned}
$$

so $A^{H} B$ is symmetric. Then, Lemma 7 implies that $\mathbf{j} B^{H} A=$ $A B^{H} \mathbf{j}$. So, according to Theorem 9

$$
A+B \mathbf{j}=\left[\begin{array}{cccc}
z_{3}^{*}-z_{1}^{*} \mathbf{j} & 2 z_{2} \mathbf{j} & z_{1}+z_{3} \mathbf{j} & 2 z_{2} \\
-2 z_{2}^{*} \mathbf{j} & z_{3}^{*}-z_{1} \mathbf{j} & -2 z_{2}^{*} & z_{1}^{*}+z_{3} \mathbf{j} \\
-z_{1}^{*}+z_{3}^{*} \mathbf{j} & 2 z_{2} & z_{3}+z_{1} \mathbf{j} & 2 z_{2} \mathbf{j} \\
-2 z_{2}^{*} & -z_{1}+z_{3}^{*} \mathbf{j} & -2 z_{2}^{*} \mathbf{j} & z_{3}+z_{1}^{*} \mathbf{j}
\end{array}\right]
$$

satisfies the orthogonality constraint $(A+\mathbf{j} B)^{Q}(A+\mathbf{j} B)=$ $2\left(\left|z_{1}\right|^{2}+4\left|z_{2}\right|^{2}+\left|z_{3}\right|^{2}\right) I$. Hence, $A+B \mathbf{j}$ is a $\operatorname{QOD}(4,4 ; 2,8,2)$ with linear processing on the complex variables $z_{1}, z_{2}, z_{3}$. It is possible to rewrite this example using quaternion variables $\mathbf{a}, \mathbf{b}$, where (for example) $\mathbf{a}=z_{1}+z_{3} \mathbf{j}$ and $2 z_{2}=\mathbf{b}-\mathbf{i b i}$. We omit the details for brevity.

We note that in applying Theorem 9 (similarly Corollary 10), $A$ and $B$ need not contain the same variables, since we can simply take the union of their variables as the set of possible variables and set certain coefficients $s_{h}$ and $t_{h}$ to be zero as necessary. We illustrate with the following examples.

Example 13: We apply Theorem 9 to the following complex orthogonal designs on complex variables $z_{1}, z_{2}$ : $A=\left[\begin{array}{cc}z_{1} & 0 \\ 0 & -z_{1}^{*}\end{array}\right]$ and $B=\left[\begin{array}{cc}z_{2} & 0 \\ 0 & -z_{2}^{*}\end{array}\right]$. We can say that $A$ is a $\operatorname{COD}(2,2 ; 1,0)$ and $B$ is a $\operatorname{COD}(2,2 ; 0,1)$, with both designs defined on the complex variables $\left\{z_{1}, z_{2}\right\}$. It is straightforward to verify that $A^{H} B$ is symmetric. Then, $A+B \mathbf{j}$ gives $\left[\begin{array}{cc}z_{1}+z_{2} \mathbf{j} & 0 \\ 0 & -z_{1}^{*}-z_{2}^{*} \mathbf{j}\end{array}\right]$, which is a $\operatorname{QOD}(2,2 ; 1,1)$ with linear processing on complex variables $z_{1}, z_{2}$. Writing $\mathbf{a}=z_{1}+z_{2} \mathbf{j}$ we have $A+B \mathbf{j}=\left[\begin{array}{cc}\mathbf{a} & 0 \\ 0 & \mathbf{j a j}\end{array}\right]$, which shows that $A+B \mathbf{j}$ can also be considered a $\mathrm{QOD}$ on the quaternion variable a.

Example 14: We apply Corollary 10 to the CODs $A$ and $B$ from Example 8. We can denote $A$ as a $\operatorname{COD}(2,2 ; 1,1,0,0)$ and $B$ as a $\operatorname{COD}(2,2 ; 0,0,1,1)$ on the real variables $\left\{x_{1}, x_{2}, x_{3}, x_{4}\right\}$. Then, $A+B \mathbf{j}=\left[\begin{array}{cc}x_{1}+x_{3} \mathbf{j} & x_{2} \mathbf{i}+x_{4} \mathbf{k} \\ x_{2} \mathbf{i}+x_{4} \mathbf{k} & x_{1}+x_{3} \mathbf{j}\end{array}\right]$. It can be shown that $(A+B \mathbf{j})^{Q}(A+B \mathbf{j})=\sum_{i=1}^{4} x_{i}^{2} I$, so that $A+B \mathbf{j}$ is a $\operatorname{QOD}(2 ; 1,1,1,1)$ with linear processing on real variables $x_{1}, x_{2}, x_{3}, x_{4}$. Alternatively, writing $\mathbf{a}=x_{1}+x_{2} \mathbf{i}+x_{3} \mathbf{j}+x_{4} \mathbf{k}$, we have $A+B \mathbf{j}=$ $(1 / 2)\left[\begin{array}{ll}\mathbf{a}-\mathbf{j a j} & \mathbf{a}+\mathbf{j a j} \\ \mathbf{a}+\mathbf{j a j} & \mathbf{a}-\mathbf{j a j}\end{array}\right]$, which shows that our matrix can also be considered a QOD with linear processing on the quaternion variable $\mathbf{a}$.

The QODs produced in Examples 11, 12, and 14 clearly contain no zero entries. As space-time block codes with no (or few) zero entries have practical advantages when used in signal processing [17], we expect that QODs with no zero entries will have similar advantages. However, the quaternion case is complicated because although each entry in the above examples is nonzero, it is not the case that each entry is a full quaternion variable $\mathbf{a}=a_{1}+a_{2} \mathbf{i}+a_{3} \mathbf{j}+a_{4} \mathbf{k}$, with all nonzero real components $a_{h}$. It would likely be useful if all entries were nonzero in at least one of the polarization planes. Further work must be completed to determine any practical implementation advantages of these designs with no zero entries.

\section{QUATERNION CONSTRUCTION TECHNIQUES}

The goal of this section is to develop theory that can be used to construct QODs without a reliance on ODs or CODs. Although the OD- and COD-dependent construction techniques of Section II provide insight into quaternion orthogonality and contribute to a library of examples of QODs, the resulting 
QODs are necessarily limited by the structure of the OD and COD building blocks. In practical applications, the goal will be to maximize the rate and minimize the decoding delay of the QODs. By relying on CODs in constructions, we may be limiting the rate of the resulting QODs due to limitations of the CODs. Therefore, it is of practical importance to develop QOD construction techniques that do not rely on existing ODs or CODs.

This section also differs from Section II in that the focus is on QODs that are defined naturally on quaternion variables, as opposed to QODs that are defined naturally on real or complex variables and then manipulated to be written using quaternion variables. QODs on more than one quaternion variable appear to be the most difficult type of QODs to construct.

In Section III-A, we define quaternion-commutivity as a way to overcome certain of the difficulties presented by the non-commutivity of the quaternions. In Section III-B, we define quaternion amicable designs, which we expect to be building blocks in future QOD constructions. In Section III-C, we investigate certain conditions required to build $2 \times 2$ QODs on two quaternion variables.

\section{A. Quaternion-Commuting Variables}

The noncommutivity of the quaternions is clearly a hurdle in developing construction techniques for QODs, as it prevents simple generalizations of known construction techniques for ODs and CODs. In this subsection, we investigate a possible condition that would allow us to keep the benefits of including the $\mathbf{j}$ and $\mathbf{k}$ components of the quaternion variables while restricting the quaternion variables so that they behave more like commuting variables.

Definition 15: Let $\mathbf{a}$ and $\mathbf{b}$ be two quaternion variables. Then, $\mathbf{a}$ and $\mathbf{b}$ are said to quaternion-commute when $\mathbf{a b} \mathbf{b}^{Q}=\mathbf{b a}^{Q}$ and/or $\mathbf{a}^{Q} \mathbf{b}=\mathbf{b}^{Q} \mathbf{a}$.

Our next example shows the utility of this definition as applied to two quaternion variables.

Example 16: Given the success of the Alamouti code represented by the $\operatorname{COD} Z=\left[\begin{array}{cc}z_{1} & z_{2} \\ z_{2}^{*} & -z_{1}^{*}\end{array}\right]$, it is natural to start with $A=\left[\begin{array}{cc}\mathbf{a} & \mathbf{b} \\ \mathbf{b}^{Q} & -\mathbf{a}^{Q}\end{array}\right]$ and look for modification that would convert $A$ into a $\mathrm{QOD}$ on quaternion variables $\mathbf{a}, \mathbf{b}$. Of course, $A$ only satisfies the orthogonality constraint if $\mathbf{a}$ and $\mathbf{b}$ commute. However, the modification $A=\left[\begin{array}{cc}\mathbf{a} & \mathbf{b} \\ \mathbf{b} & -\mathbf{a}\end{array}\right]$ satisfies the orthogonality constraint if $\mathbf{a}$ and $\mathbf{b}$ are chosen to be quaternion-commuting variables. In this case

$$
\begin{aligned}
A A^{Q} & =\left[\begin{array}{cc}
\mathbf{a} & \mathbf{b} \\
\mathbf{b} & -\mathbf{a}
\end{array}\right]\left[\begin{array}{cc}
\mathbf{a}^{Q} & \mathbf{b}^{Q} \\
\mathbf{b}^{Q} & -\mathbf{a}^{Q}
\end{array}\right] \\
& =\left[\begin{array}{cc}
\mathbf{a} \mathbf{a}^{Q}+\mathbf{b} \mathbf{b}^{Q} & \mathbf{a} \mathbf{b}^{Q}-\mathbf{b} \mathbf{a}^{Q} \\
\mathbf{b} \mathbf{a}^{Q}-\mathbf{a} \mathbf{b}^{Q} & \mathbf{b b}^{Q}+\mathbf{a} \mathbf{a}^{Q}
\end{array}\right] \\
& =\left(\mathbf{a} \mathbf{a}^{Q}+\mathbf{b} \mathbf{b}^{Q}\right) I
\end{aligned}
$$

and similarly for $A^{Q} A$. Thus, $A$ is a QOD on the quaternioncommuting variables $\mathbf{a}$ and $\mathbf{b}$.
We now address the natural question of which quaternion variables quaternion-commute. To do this, we must introduce some notation:

Definition 17: Let $\mathbf{a}, \mathbf{b}$ be quaternion variables with $\mathbf{a}=$ $a_{1}+a_{2} \mathbf{i}+a_{3} \mathbf{j}+a_{4} \mathbf{k}$ and $\mathbf{b}=b_{1}+b_{2} \mathbf{i}+b_{3} \mathbf{j}+b_{4} \mathbf{k}$, where $a_{h}, b_{h}$ are real variables for $1 \leq h \leq 4$. Then, we denote the product $\mathbf{a b}^{Q}=\sigma(\mathbf{a}, \mathbf{b})=\sigma_{1}+\sigma_{2} \mathbf{i}+\sigma_{3} \mathbf{j}+\sigma_{4} \mathbf{k}$, where

$$
\begin{aligned}
\sigma_{1} & =\sigma_{1}(\mathbf{a}, \mathbf{b})=a_{1} b_{1}+a_{2} b_{2}+a_{3} b_{3}+a_{4} b_{4} \\
\sigma_{2} & =\sigma_{2}(\mathbf{a}, \mathbf{b})=-a_{1} b_{2}+a_{2} b_{1}-a_{3} b_{4}+a_{4} b_{3} \\
\sigma_{3} & =\sigma_{3}(\mathbf{a}, \mathbf{b})=-a_{1} b_{3}+a_{2} b_{4}+a_{3} b_{1}-a_{4} b_{2} \\
\sigma_{4} & =\sigma_{4}(\mathbf{a}, \mathbf{b})=-a_{1} b_{4}-a_{2} b_{3}+a_{3} b_{2}+a_{4} b_{1} .
\end{aligned}
$$

Similarly, $\mathbf{a}^{Q} \mathbf{b}=\tau(\mathbf{a}, \mathbf{b})=\tau_{1}+\tau_{2} \mathbf{i}+\tau_{3} \mathbf{j}+\tau_{4} \mathbf{k}$, where

$$
\begin{aligned}
& \tau_{1}=\tau_{1}(\mathbf{a}, \mathbf{b})=a_{1} b_{1}+a_{2} b_{2}+a_{3} b_{3}+a_{4} b_{4} \\
& \tau_{2}=\tau_{2}(\mathbf{a}, \mathbf{b})=a_{1} b_{2}-a_{2} b_{1}-a_{3} b_{4}+a_{4} b_{3} \\
& \tau_{3}=\tau_{3}(\mathbf{a}, \mathbf{b})=a_{1} b_{3}+a_{2} b_{4}-a_{3} b_{1}-a_{4} b_{2} \\
& \tau_{4}=\tau_{4}(\mathbf{a}, \mathbf{b})=a_{1} b_{4}-a_{2} b_{3}+a_{3} b_{2}-a_{4} b_{1} .
\end{aligned}
$$

Lemma 18: Two quaternion variables $\mathbf{a}$ and $\mathbf{b}$ quaternioncommute with $\mathbf{a b}^{Q}=\mathbf{b a}^{Q}$ (and/or $\mathbf{a}^{Q} \mathbf{b}=\mathbf{b}^{Q} \mathbf{a}$ ) if and only if $\mathbf{a b}^{Q}$ is real (and/or $\mathbf{a}^{Q} \mathbf{b}$ is real).

Proof: Using the $\sigma$ notation developed in Definition 17, we see that just as $\mathbf{a b}^{Q}=\sigma_{1}+\sigma_{2} \mathbf{i}+\sigma_{3} \mathbf{j}+\sigma_{4} \mathbf{k}$, we have $\mathbf{b a}^{Q}=\left(\mathbf{a b}^{Q}\right)^{Q}=\sigma_{1}-\sigma_{2} \mathbf{i}-\sigma_{3} \mathbf{j}-\sigma_{4} \mathbf{k}$. Thus

$$
\begin{aligned}
& \mathbf{a b}^{Q}=\mathbf{b a}^{Q} \Longleftrightarrow \mathbf{a b}^{Q}-\mathbf{b a}^{Q}=0 \\
& \quad \Longleftrightarrow\left(\sigma_{1}+\sigma_{2} \mathbf{i}+\sigma_{3} \mathbf{j}+\sigma_{4} \mathbf{k}\right)-\left(\sigma_{1}-\sigma_{2} \mathbf{i}-\sigma_{3} \mathbf{j}-\sigma_{4} \mathbf{k}\right) \\
& \quad=0 \\
& \quad \Longleftrightarrow 2\left(\sigma_{2} \mathbf{i}+\sigma_{3} \mathbf{j}+\sigma_{4} \mathbf{k}\right)=0 \\
& \quad \Longleftrightarrow \sigma_{2}=\sigma_{3}=\sigma_{4}=0 .
\end{aligned}
$$

Hence, $\mathbf{a} \mathbf{b}^{Q}$ is real. The case for $\mathbf{a}^{Q} \mathbf{b}=\mathbf{b}^{Q} \mathbf{a}$ follows similarly with $\mathbf{a}^{Q} \mathbf{b}=\mathbf{b}^{Q} \mathbf{a} \Longleftrightarrow \tau_{2}=\tau_{3}=\tau_{4}=0$. (We observe that for both $\mathbf{a} \mathbf{b}^{Q}=\mathbf{b} \mathbf{a}^{Q}$ and $\mathbf{a}^{Q} \mathbf{b}=\mathbf{b}^{Q} \mathbf{a}$ to hold, we require $a_{\ell} b_{m}=b_{\ell} a_{m}$, for all $\ell \neq m, \ell, m=1,2,3,4$.)

Example 19: Suppose $\mathbf{a}$ and $\mathbf{b}$ are quaternion-commuting variables such that $\mathbf{a b}^{Q}=\mathbf{b a}^{Q}$ is real. Then, $D=\left[\begin{array}{cc}\mathbf{a} & \mathbf{j b} \\ \mathbf{i b} & -\mathrm{ka}\end{array}\right]$ is a QOD on these specific quaternion-commuting variables $\mathbf{a}$ and $\mathbf{b}$. This follows as

$$
\begin{aligned}
D D^{Q} & =\left[\begin{array}{cc}
\mathbf{a} & \mathbf{j b} \\
\mathbf{i b} & -\mathbf{k} \mathbf{a}
\end{array}\right]\left[\begin{array}{cc}
\mathbf{a}^{Q} & -\mathbf{b}^{Q} \mathbf{i} \\
-\mathbf{b}^{Q} \mathbf{j} & \mathbf{a}^{Q} \mathbf{k}
\end{array}\right] \\
& =\left[\begin{array}{cc}
\mathbf{a} \mathbf{a}^{Q}+\mathbf{b b}^{Q} & -\mathbf{a b} \mathbf{b}^{Q} \mathbf{i}+\mathbf{j} \mathbf{b} \mathbf{a}^{Q} \mathbf{k} \\
\mathbf{i} \mathbf{b} \mathbf{a}^{Q}+\mathbf{k} \mathbf{a} \mathbf{b}^{Q} \mathbf{j} & \mathbf{a} \mathbf{a}^{Q}+\mathbf{b b}^{Q}
\end{array}\right] \\
& =\left(|\mathbf{a}|^{2}+|\mathbf{b}|^{2}\right) I_{2} .
\end{aligned}
$$

It also holds that $D^{Q} D=\left(\mathbf{a}^{Q} \mathbf{a}+\mathbf{b}^{Q} \mathbf{b}\right) I_{2}=\left(|\mathbf{a}|^{2}+|\mathbf{b}|^{2}\right) I_{2}$. We will later show in condition 2) of Example 24 that the proposed matrix $D$ is also a QOD if the quaternion variables $\mathbf{a}, \mathbf{b}$ are instead restricted so that only $\sigma_{3}=0$, where $\sigma_{3}=\sigma_{3}(\mathbf{a}, \mathbf{b})$ is as defined in Definition 17.

We have demonstrated that quaternion-commuting variables can be used to build QODs, however Lemma 18 shows that 
quaternion-commuting variables are subject to very strong restrictions. Therefore, this construction method is likely to be difficult for sizes greater than 2, as these would require more than two mutually quaternion-commuting variables. However, this notion of quaternion-commuting variables might be useful in conjunction with other conditions on certain of the quaternion variables.

\section{B. Amicable Designs}

Real amicable designs were defined over a quarter of a century ago [15], [16], [19], [20], and yet many questions concerning these designs, as well as complex amicable designs, remain unsolved.

Definition 20: Two square real orthogonal designs $A$ and $B$ are said to be amicable if $A^{T} B=B^{T} A$ and $A B^{T}=B A^{T}$. Two generalized complex orthogonal designs $A$ and $B$ are said to be complex amicable if $A B^{H}=B A^{H}$ and/or $A^{H} B=$ $B^{H} A$.

Example 21: One can confirm that $A=\left[\begin{array}{cc}x_{1} & x_{2} \\ -\mathbf{i} x_{2} & \mathbf{i} x_{1}\end{array}\right]$ and $B=\left[\begin{array}{cc}x_{3} & x_{4} \\ \mathbf{i} x_{4} & -\mathbf{i} x_{3}\end{array}\right]$ are CODs on real variables $x_{1}, x_{2}, x_{3}, x_{4}$. Furthermore, $A$ and $B$ are complex amicable designs. This can be seen by noting that

$$
A^{H} B=\left[\begin{array}{cc}
x_{1} x_{3}-x_{2} x_{4} & x_{1} x_{4}+x_{2} x_{3} \\
x_{2} x_{3}+x_{1} x_{4} & -x_{1} x_{3}+x_{2} x_{4}
\end{array}\right]
$$

is real and symmetric. This implies that $A^{H} B=\left(A^{H} B\right)^{T}=$ $\left(A^{H} B\right)^{H}=B^{H} A$. So, $A^{H} B=B^{H} A$. One can also verify that $A B^{H}=B A^{H}$.

Real amicable designs have been used to build complex orthogonal designs, and there has been a renewed interest in amicable designs due to the discovery that they can be utilized in space-time coding [8], [9], [21], [23]. Motivated by the utility of the real and complex cases, we propose a definition for quaternion amicable designs and investigate their existence in the $2 \times 2$ case.

Definition 22: Let $A$ and $B$ be quaternion orthogonal designs. If $A B^{Q}=B A^{Q}$, then say $A$ and $B$ are quaternion amicable designs.

Lemma 23: Suppose $\mathbf{a}, \mathbf{b}, \mathbf{x}$, and $\mathbf{y}$ are quaternion variables such that

$$
\begin{aligned}
\mathbf{a x} \mathbf{x}^{Q} & =\mathbf{x a}^{Q} \\
\mathbf{b} \mathbf{y}^{Q} & =\mathbf{y b}^{Q} \\
\tau_{2}(\mathbf{a}, \mathbf{b}) & =a_{1} b_{2}-a_{2} b_{1}-a_{3} b_{4}+a_{4} b_{3}=0 \\
\tau_{2}(\mathbf{x}, \mathbf{y}) & =x_{1} y_{2}-x_{2} y_{1}-x_{3} y_{4}+x_{4} y_{3}=0 \\
\tau_{2}(\mathbf{a}, \mathbf{y}) & =a_{1} y_{2}-a_{2} y_{1}-a_{3} y_{4}+a_{4} y_{3}=0 \\
\tau_{2}(\mathbf{b}, \mathbf{x}) & =b_{1} x_{2}-b_{2} x_{1}-b_{3} x_{4}+b_{4} x_{3}=0 .
\end{aligned}
$$

Then, the matrices

$$
A=\left[\begin{array}{cc}
\mathbf{a} & \mathbf{b j} \\
\mathbf{b i} & -\mathbf{a k}
\end{array}\right] \text { and } B=\left[\begin{array}{cc}
\mathbf{x} & -\mathbf{y j} \\
\mathbf{y i} & \mathbf{x k}
\end{array}\right]
$$

are QODs on the specific quaternion variables $\mathbf{a}, \mathbf{b}, \mathbf{x}, \mathbf{y}$. (Given $\tau_{2}(\mathbf{a}, \mathbf{b})=0, A$ is orthogonal; given $\tau_{2}(\mathbf{x}, \mathbf{y})=0, B$ is orthogonal.) Also, $A B^{Q}=B A^{Q}$ so $A$ and $B$ are quaternion amicable designs.

Proof: Let aiy ${ }^{Q}=\alpha_{1}+\alpha_{2} \mathbf{i}+\alpha_{3} \mathbf{j}+\alpha_{4} \mathbf{k}$. Then $\left(\text { aiy }^{Q}\right)^{Q}=$ $\alpha_{1}-\alpha_{2} \mathbf{i}-\alpha_{3} \mathbf{j}-\alpha_{4} \mathbf{k}$. Now, $\mathbf{y i a}^{Q}=-\left(\text { aiy }^{Q}\right)^{Q}$. Hence, yia $^{Q}=$ $-\alpha_{1}+\alpha_{2} \mathbf{i}+\alpha_{3} \mathbf{j}+\alpha_{4} \mathbf{k}$. But $\alpha_{1}=a_{1} y_{2}-a_{2} y_{1}-a_{3} y_{4}+a_{4} y_{3}=$ $\tau_{2}(a, y)=0$. So aiy ${ }^{Q}=$ yia $^{Q}$. Likewise, it can be shown that $\mathbf{b i x}^{Q}=\mathbf{x i b}^{Q}$, given $\tau_{2}(b, x)=0$. Then,

$$
\begin{aligned}
& A B^{Q}=\left[\begin{array}{cc}
\mathbf{a} & \mathbf{b j} \\
\mathbf{b i} & -\mathbf{a k}
\end{array}\right]\left[\begin{array}{cc}
\mathbf{x}^{Q} & -\mathbf{i} \mathbf{y}^{Q} \\
\mathbf{j} \mathbf{y}^{Q} & -\mathbf{k} \mathbf{x}^{Q}
\end{array}\right] \\
& =\left[\begin{array}{cc}
\mathbf{a x}^{Q}-\mathbf{b y}^{Q} & -\mathbf{a i y}^{Q}-\mathbf{b i x} \mathbf{x}^{Q} \\
\mathbf{b i x} \mathbf{x}^{Q}+\mathbf{a i y}^{Q} & \mathbf{b y} \mathbf{y}^{Q}-\mathbf{a x}^{Q}
\end{array}\right] \\
& =\left[\begin{array}{cc}
\mathbf{x a}^{Q}-\mathbf{y b}^{Q} & -\mathbf{y i a}^{Q}-\mathbf{x i b}^{Q} \\
\mathbf{x i b}^{Q}+\mathbf{y i a}^{Q} & \mathbf{y b}^{Q}-\mathbf{x a}^{Q}
\end{array}\right] \\
& =\left[\begin{array}{cc}
\mathbf{x} & -\mathbf{y j} \\
\mathbf{y i} & \mathbf{x k}
\end{array}\right]\left[\begin{array}{cc}
\mathbf{a}^{Q} & -\mathbf{i b} \mathbf{b}^{Q} \\
-\mathbf{j b}^{Q} & \mathbf{k a}^{Q}
\end{array}\right] \\
& =B A^{Q} \text {. }
\end{aligned}
$$

Thus, we have provided some conditions (albeit restrictive) under which $2 \times 2$ quaternion amicable designs exist. Many open questions remain concerning the construction and theory of quaternion amicable designs (as well as for real and complex amicable designs). A question of particular interest is to determine if small examples of quaternion amicable designs can be used to build larger QODs.

\section{Size-Two Quaternion Orthogonal Designs}

Thus far, it has proven difficult to construct QODs on more than one quaternion variable. In order to build such designs without the use of ODs or CODs, we must develop an understanding of the forced constraints on the quaternion variables appearing in these designs. In this subsection, we study constraints under which we can design $2 \times 2$ QODs on two quaternion variables.

Example 24: Consider the matrix $D=\left[\begin{array}{cc}\mathbf{a} & t \mathbf{b} \\ s \mathbf{b} & -u \mathbf{a}\end{array}\right]$, with $s, t, u \in\{ \pm \mathbf{i}, \pm \mathbf{j}, \pm \mathbf{k}\}$ and quaternion variables $\mathbf{a}$ and $\mathbf{b}$. Then

$$
\begin{aligned}
D D^{Q} & =\left[\begin{array}{cc}
\mathbf{a} & t \mathbf{b} \\
s \mathbf{b} & -u \mathbf{a}
\end{array}\right]\left[\begin{array}{cc}
\mathbf{a}^{Q} & -\mathbf{b}^{Q} s \\
-\mathbf{b}^{Q} t & \mathbf{a}^{Q} u
\end{array}\right] \\
& =\left[\begin{array}{cc}
\mathbf{a a}^{Q}+\mathbf{b b}^{Q} & -\mathbf{a b}^{Q} s+t \mathbf{b a}^{Q} u \\
s \mathbf{b a}^{Q}+u \mathbf{a} \mathbf{b}^{Q} t & \mathbf{a a}^{Q}+\mathbf{b b}^{Q}
\end{array}\right] .
\end{aligned}
$$

For this to be a $\operatorname{QOD}(2 ; 1,1)$, we require the off-diagonal elements to equal 0 . We observe

$$
\begin{aligned}
\left(-\mathbf{a} \mathbf{b}^{Q} s+t \mathbf{b} \mathbf{a}^{Q} u\right)^{Q} & =\left(-\mathbf{a b}^{Q} s\right)^{Q}+\left(t \mathbf{b a}^{Q} u\right)^{Q} \\
& =s \mathbf{b a}^{Q}+u \mathbf{a} \mathbf{b}^{Q} t .
\end{aligned}
$$

Thus, if one of the diagonal elements equals 0 , it follows that the other element is 0 . An analysis shows that for $s \mathbf{b a}^{Q}+u \mathbf{a b}{ }^{Q} t=$ 0 to hold for arbitrary quaternion variables $\mathbf{a}, \mathbf{b}$, it would be 
required that the following four equations are simultaneously satisfied:

$$
\begin{aligned}
s+u t & =0 \\
s \mathbf{i} & =u \mathbf{i} t \\
s \mathbf{j} & =u \mathbf{j} t \\
s \mathbf{k} & =u \mathbf{k} t .
\end{aligned}
$$

However, a lengthy analysis shows that it is not possible to simultaneously satisfy the above four equations, and therefore we cannot find solutions to $s \mathbf{b a}^{Q}+u \mathbf{a b} \mathbf{b}^{Q} t=0$ that hold for arbitrary quaternion variables $\mathbf{a}, \mathbf{b}$. Nevertheless, by imposing certain restrictions on the quaternion variables $\mathbf{a}, \mathbf{b}$, we can still obtain conditions under which $s \mathbf{b a}^{Q}+u \mathbf{a b}{ }^{Q} t=0$ so that the proposed $D$ is a QOD. The following are conditions under which $s \mathbf{b a}^{Q}+u \mathbf{a b}^{Q} t=0$, where the $\sigma_{h}=\sigma_{h}(\mathbf{a}, \mathbf{b})$ are the components of $\mathbf{a b}^{Q}$ as given in Definition 17:

1) $s=\mathbf{i}, t= \pm \mathbf{k}, u=\mp \mathbf{j}, \sigma_{4}=0$;

2) $s=\mathbf{i}, t= \pm \mathbf{j}, u= \pm \mathbf{k}, \sigma_{3}=0$;

3) $s=\mathbf{j}, t= \pm \mathbf{k}, u= \pm \mathbf{i}, \sigma_{4}=0$;

4) $s=\mathbf{j}, t= \pm \mathbf{i}, u=\mp \mathbf{k}, \sigma_{2}=0$;

5) $s=\mathbf{k}, t= \pm \mathbf{j}, u=\mp \mathbf{i}, \sigma_{3}=0$;

6) $s=\mathbf{k}, t= \pm \mathbf{i}, u= \pm \mathbf{j}, \sigma_{2}=0$.

Hence, given quaternion variables and coefficients that satisfy one of the above six sets of constraints, we can form a QOD of the form $D=\left[\begin{array}{cc}\mathbf{a} & t \mathbf{b} \\ s \mathbf{b} & -u \mathbf{a}\end{array}\right]$. We note that solution 2) was previously presented by Finlayson, Seberry, Wysocki, and Xia [7]. We have deliberately chosen to consider only solutions where $s$ has positive sign, due to our Lemma 25 below.

Lemma 25: Let $D$ be the matrix $\left[\begin{array}{cc}\mathbf{a} & t \mathbf{b} \\ s \mathbf{b} & -u \mathbf{a}\end{array}\right]$, and let $\mathbf{a b}{ }^{Q}=$ $\sigma_{1}+\sigma_{2} \mathbf{i}+\sigma_{3} \mathbf{j}+\sigma_{4} \mathbf{k}$. Given the condition that $\sigma_{p}=0$, for some $p=1,2,3,4$, if the use of $s, t, u$, where $s, t, u \in\{ \pm \mathbf{i}, \pm \mathbf{j}, \pm \mathbf{k}\}$ implies that $D$ is a QOD on quaternion variables $\mathbf{a}$ and $\mathbf{b}$, then the use of $-s, t,-u$ and $-s,-t, u$ also implies that $D$ is a QOD.

Lemma 25 is motivated by the preceding Example 24, and its proof is straightforward. Lemma 25 and Example 24 conspicuously do not consider scaling the $(1,1)$ position with an element from $\{ \pm \mathbf{i}, \pm \mathbf{j}, \pm \mathbf{k}\}$. We now consider this situation:

Example 26: Consider the matrix $D^{\prime}=\left[\begin{array}{cc}r \mathbf{a} & t \mathbf{b} \\ s \mathbf{b} & -u \mathbf{a}\end{array}\right]$, with $r, s, t, u \in\{ \pm \mathbf{i}, \pm \mathbf{j}, \pm \mathbf{k}\}$. Then

$$
\begin{aligned}
D^{\prime} D^{\prime Q} & =\left[\begin{array}{cc}
r \mathbf{a} & t \mathbf{b} \\
s \mathbf{b} & -u \mathbf{a}
\end{array}\right]\left[\begin{array}{cc}
-\mathbf{a}^{Q} r & -\mathbf{b}^{Q} s \\
-\mathbf{b}^{Q} t & \mathbf{a}^{Q} u
\end{array}\right] \\
& =\left[\begin{array}{cc}
\mathbf{a a}^{Q}+\mathbf{b b}^{Q} & -r \mathbf{a} \mathbf{b}^{Q} s+t \mathbf{b a}^{Q} u \\
-s \mathbf{b a}^{Q} r+u \mathbf{a} \mathbf{b}^{Q} t & \mathbf{a a}^{Q}+\mathbf{b b}^{Q}
\end{array}\right] .
\end{aligned}
$$

As before, for this to be a $\operatorname{QOD}(2 ; 1,1)$, the off-diagonal elements must equal zero. We can achieve this by placing conditions on $\mathbf{a}$ and $\mathbf{b}$ through restricting the terms $\sigma_{h}(\mathbf{a}, \mathbf{b})$ as follows:

1) $r=s, \sigma_{1}=0$;

2) $r=\mathbf{i}, s=\mathbf{j}, t=-\mathbf{j}, u=\mathbf{i}, \sigma_{4}=0$;

3) $r=\mathbf{i}, s=\mathbf{k}, t=-\mathbf{k}, u=\mathbf{i}, \sigma_{3}=0$;

4) $r=\mathbf{j}, s=\mathbf{k}, t=-\mathbf{k}, u=\mathbf{j}, \sigma_{2}=0$.
In this example, we have limited our list to cases with positive $r$ and $s$ due to the following Lemma 27; the proof of this lemma is straightforward.

Lemma 27: Let $D$ be the matrix $\left[\begin{array}{cc}r \mathbf{a} & -s \mathbf{b} \\ s \mathbf{b} & -r \mathbf{a}\end{array}\right]$, and let $\mathbf{a b}^{Q}=$ $\sigma_{1}+\sigma_{2} \mathbf{i}+\sigma_{3} \mathbf{j}+\sigma_{4} \mathbf{k}$. Given the condition $\sigma_{h}=0$ for some $1 \leq$ $h \leq 4$, if the use of $r, s$ imply that $D$ is a QOD on quaternion variables $\mathbf{a}$ and $\mathbf{b}$, then the use of the $r^{\prime}=-r, s^{\prime}=s ; r^{\prime}=r$, $s^{\prime}=-s ; r^{\prime}=-r, s^{\prime}=-s$ and $r^{\prime}=s, s^{\prime}=r$ also imply that $D$ is a QOD.

To see the distinction between QODs of the types introduced in Examples 24 and 26, let $D$ be the $\operatorname{QOD}(2 ; 1,1)\left[\begin{array}{cc}r \mathbf{a} & t \mathbf{b} \\ s \mathbf{b} & -u \mathbf{a}\end{array}\right]$ for some $r, s, t, u \in\{ \pm \mathbf{i}, \pm \mathbf{j}, \pm \mathbf{k}\}$. We can write $D \stackrel{=}{=}$ $r\left[\begin{array}{cc}\mathbf{a} & t^{\prime} \mathbf{b} \\ s^{\prime} \mathbf{b} & -u^{\prime} \mathbf{a}\end{array}\right]=r D^{\prime}$, where $t^{\prime}, s^{\prime}, u^{\prime} \in\{ \pm \mathbf{i}, \pm \mathbf{j}, \pm \mathbf{k}\}$. However, the conditions required to make $D$ a QOD are not consistent with the conditions required for $D^{\prime}$ to be a QOD. Therefore, the designs introduced in Examples 24 and 26 are not equivalent.

A very similar analysis can be done using quaternion premultipliers on the right, rather than the left, of the entries of a $\operatorname{QOD}(2,2 ; 1,1)$. Despite the differences due to the noncommutivity of the quaternions, the constraints are similar. We omit the details for brevity.

\section{Simulation Results}

In this section, we provide some numerical results concerning the implementation of a quaternion orthogonal design as an orthogonal space-time-polarization block code (OSTPBC). As with traditional complex orthogonal space-time block codes [18], OSTPBCs can be used in scenarios involving transmission of complex signals from several transmit antennas to one or more receive antennas, as shown in Fig. 1. The only difference is that with OSTPBCs the antennas are dual polarized antennas. Hence, in case of a single receive antenna and $N$ transmit antennas, each channel is described by the channel gain matrix $H^{(m)}=\left[\begin{array}{ll}h_{11}^{(m)} & h_{12}^{(m)} \\ h_{21}^{(m)} & h_{22}^{(m)}\end{array}\right]$, for $m=1,2, \ldots, N$, where $h_{11}$ and $h_{22}$ are complex channel gains for signals received with the same polarization as they were transmitted, and $h_{12}$ and $h_{21}$ are complex channel gains for a cross-polar scatter, i.e., signals received with different polarization from that at which it was transmitted due to scatter, reflections, and polarization twist between the transmit and receive antennas.

Introducing representation of a quaternion variable $s=z_{1}+$ $z_{2} \mathbf{j}$ as $s=\left[z_{1}, z_{2}\right]$, transmission of the dual-polarized signal through the channel described by a matrix $H$ can be modeled as a product $s H$. Hence, the received quaternion signal $r$ is given by

$$
\begin{aligned}
r & =s H+n \\
& =\left[z_{1}, z_{2}\right] H+\left[n_{1}, n_{2}\right] \\
& =\left[z_{1} h_{11}+z_{2} h_{21}, z_{1} h_{12}+z_{2} h_{22}\right]+\left[n_{1}, n_{2}\right] \\
& =\left(z_{1} h_{11}+z_{2} h_{21}\right)+\left(z_{1} h_{12}+z_{2} h_{22}\right) \mathbf{j}+n_{1}+n_{2} \mathbf{j}
\end{aligned}
$$

where $n_{1}, n_{2}$ are complex additive noises being the independent identically distributed (i.i.d.) zero-mean two-dimensional Gaussian random variables with identical variance per dimension. 


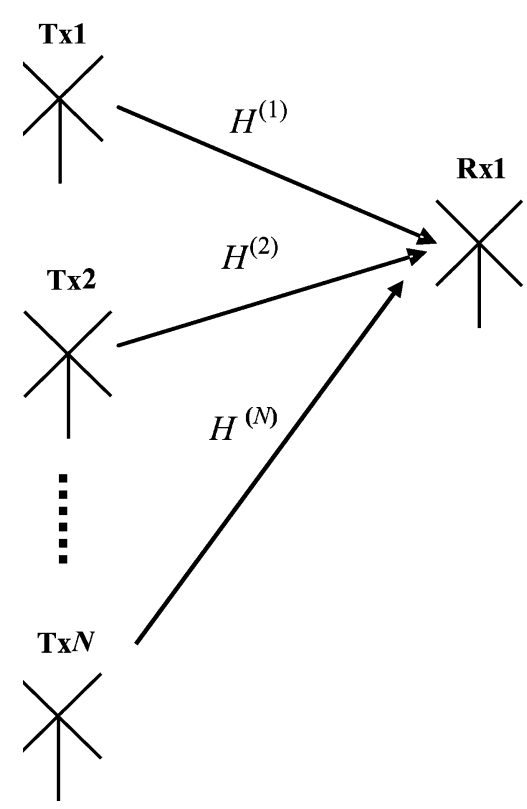

Fig. 1. Considered transmission system utilizing $N$ dual-polarized transmit antennas $T x 1, \ldots, T x N$, and a single receive dual-polarized antenna.

In this paper, we will consider only the case of a single receive antenna but all considerations can be extended to the case of multiple receive antennas. If an OSTPBC $Q$ for $N$ transmit antennas is used, the received dual-polarized signal vector $R$ can be considered as a quaternionic vector, and, using the same notation as above, modeled as

$$
R=Q\left[\begin{array}{c}
H^{(1)} \\
\vdots \\
H^{(N)}
\end{array}\right]+\left[\begin{array}{cc}
n_{11} & n_{12} \\
\vdots & \vdots \\
n_{N 1} & n_{N 2}
\end{array}\right]
$$

where $n_{m l} ; m=1, \ldots, N, l=1,2$ represent complex noises being the independent identically distributed (i.i.d.) zero-mean two-dimensional Gaussian random variables with identical variance per dimension.

Assuming perfect channel knowledge at the receiver, i.e., assuming that matrices $H^{(1)}, \ldots, H^{(N)}$ are known and constant for some reasonable time, as in the case of quasi-static conditions, the ML decoding rule for any OSTPBC is equivalent to finding a set of signal symbols that minimizes the norm $\| R-$ $Q H \|$, where $R$ is the received signal vector, $Q$ is the code matrix, and $H$ is the matrix of complex channel coefficients. This is equivalent to finding a set of signal symbols minimizing the squared norm, which can be expressed as

$$
\begin{aligned}
\|R-Q H\|^{2}= & \operatorname{trace}\left\{(R-Q H)^{Q}(R-Q H)\right\} \\
= & \operatorname{trace}\left\{\left(R^{Q}-H^{Q} Q^{Q}\right)(R-Q H)\right\} \\
= & \operatorname{trace}\left\{R^{Q} R-R^{Q} Q H-H^{Q} Q^{Q} R\right. \\
& \left.\quad+H^{Q} Q^{Q} Q H\right\} \\
= & \operatorname{trace}\left\{R^{Q} R\right\}-2 \operatorname{Re}\left\{\operatorname{trace}\left[R^{Q} Q H\right]\right\} \\
& +\lambda \operatorname{trace}\left\{H^{Q} H\right\}
\end{aligned}
$$

where $Q^{Q} Q=\lambda I$. Thus, for any orthogonal $Q$, the decoding rule can be decoupled.
We now provide a concrete example by implementing the QOD of order 2 originally given in Example 11 as an OSTPBC for two transmit dual-polarized antennas

$$
Q_{1}=\left[\begin{array}{cc}
z_{1}+z_{2} \mathbf{j} & z_{2}+z_{1} \mathbf{j} \\
-z_{2}^{*}+z_{1}^{*} \mathbf{j} & z_{1}^{*}-z_{2}^{*} \mathbf{j}
\end{array}\right]
$$

It can be easily noticed that $Q_{1}$ comprises two Alamouti codes [1], one used for one polarization and another one, with switched columns, used for orthogonal polarization. Using the same notation as above, $Q_{1}$ can be expressed as

$$
Q_{1}=\left[\begin{array}{cccc}
z_{1} & z_{2} & z_{2} & z_{1} \\
-z_{2}^{*} & z_{1}^{*} & z_{1}^{*} & -z_{2}^{*}
\end{array}\right]
$$

where odd columns represent signals transmitted through one polarization and even columns represent signals transmitted through the polarization orthogonal to the first one.

For a single receive dual-polarized antenna, the channels between dual-polarized transmit antennas $\mathrm{Tx} 1$ and $\mathrm{Tx} 2$, and the dual-polarized receive antenna $\mathrm{Rx} 1$ are described by their own channel gain matrices, $H^{(1)}=\left[h_{m \ell}^{(1)}\right]_{2 \times 2}$ and $H^{(2)}=\left[h_{m \ell}^{(2)}\right]_{2 \times 2}$, respectively. The received signal vector $R$ is given by

$$
R=Q_{1}\left[\begin{array}{l}
H^{(1)} \\
H^{(2)}
\end{array}\right]+\left[\begin{array}{ll}
n_{11} & n_{12} \\
n_{21} & n_{22}
\end{array}\right],
$$

where $n_{m \ell} ; m, \ell=1,2$ represent complex noises being the independent identically distributed (i.i.d.) zero-mean two dimensional Gaussian random variables with identical variance per dimension.

Utilizing the principles of quaternionic arithmetic, the decoding rule can be simplified as follows: the decoding statistic for $z_{1}$ is $\min _{z_{1} \in Z}\left|z_{1}\right|^{2}\left(\left|g_{1}\right|^{2}+\left|g_{2}\right|^{2}+\left|g_{3}\right|^{2}+\right.$ $\left.\left|g_{4}\right|^{2}\right)-2 \operatorname{Re}\left\{r_{1}^{Q} z_{1}\left(g_{1}+g_{2} \mathbf{j}\right)+r_{2}^{Q} z_{1}^{*}\left(g_{3}+g_{4} \mathbf{j}\right)\right\}, \quad$ and the decoding statistic for $z_{2}$ is

$$
\begin{aligned}
& \min _{z_{2} \in Z}\left|z_{2}\right|^{2}\left(\left|g_{1}\right|^{2}+\left|g_{2}\right|^{2}+\left|g_{3}\right|^{2}+\left|g_{4}\right|^{2}\right) \\
&-2 \operatorname{Re}\left\{r_{1}^{Q} z_{2}\left(g_{3}+g_{4} \mathbf{j}\right)-r_{2}^{Q} z_{2}^{*}\left(g_{1}+g_{4} 2 \mathbf{j}\right)\right\}
\end{aligned}
$$

where $g_{1}=h_{11}^{(1)}+h_{21}^{(2)}, g_{2}=h_{12}^{(1)}+h_{22}^{(2)}, g_{3}=h_{21}^{(1)}+h_{11}^{(2)}$, and $g_{4}=h_{22}^{(1)}+h_{12}^{(2)}$.

To assess the performance gain that can be achieved using the code $Q_{1}$ and a single receive dual-polarized antenna, the system was implemented using MATLAB. The following conditions were assumed.

- The QPSK $\{0, \pi / 2, \pi, 3 \pi / 2\}$ signal constellation was applied.

- Total transmitted power in both polarizations and through both antennas was equal to 1 , and equally distributed per antenna and per polarization. For a fair comparison, the average total received power in all systems before adding noise was set to 1 , and we used the same SNR normalization as used by Alamouti [1].

- Channel coefficient matrices, $H^{(1)}$ and $H^{(2)}$ were assumed known at the receiver and kept constant for 1000 data bits. 


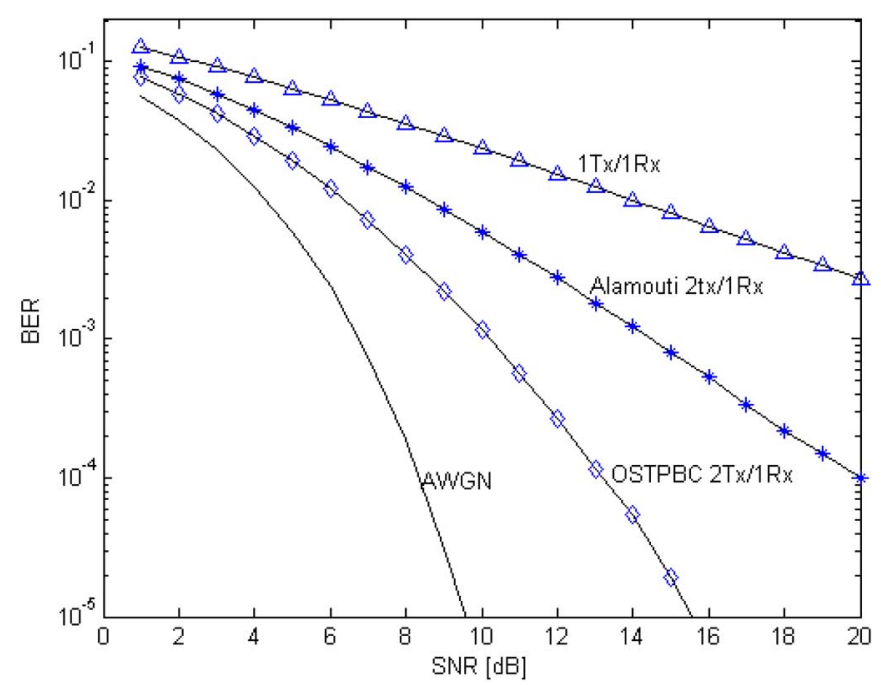

Fig. 2. Bit-error-rate (BER) performance of the developed scheme (OSTPBC) using the code $Q_{1}$ combined with QPSK modulation in a slow flat Rayleigh fading channel experiencing random cross-polar scatter compared with the Alamouti scheme and a single transmit/single receive antenna system.

- The sum of variances of all the channel coefficients was kept equal to 1 and the variance of the real and imaginary parts of a particular coefficient was identical. The variances were drawn randomly, every time the new set of coefficients was drawn.

- The channel coefficients were generated as random complex Gaussian i.i.d. variables. The additive noise was assumed to be additive white Gaussian noise added uniformly for each polarization and each real/imaginary component (a quaternion zero-mean Gaussian variable).

The simulation results are provided in Fig. 2. The evident performance improvement compared to the conventional Alamouti scheme has been achieved without a significant increase in the dimensions of the antenna system of either transmitter or receiver. These results are promising, and we expect to explore further the implementation of QODs as OSTPBCs in future work. It remains an open problem to determine if these codes can admit received signal linearization.

\section{CONCLUSION}

We have established the existence of QODs on real variables, on complex variables, and on quaternion variables. We offered construction techniques that utilize existing real and complex orthogonal designs to build QODs. These constructions provide insight into the combinatorial structure of QODs, and they are useful for building a library of QOD examples. However, by depending on existing ODs and CODs, the resulting QODs may be limited in their rate and delay. We propose that in order to generate optimal QODs, we must develop construction techniques that do not limit the QODs through imposing structure based on ODs or CODs. To work towards the goal of developing QODs (specifically QODs on quaternion variables) without reliance on ODs or CODs, we defined and explored the concepts of quaternion-commutivity and quaternion amicability. We hope these concepts will stimulate further work in this area. Our work towards building $2 \times 2$ QODs on two quaternion variables illu- minates some challenges in constructing these designs. Handling QODs on multiple quaternion variables is more difficult than handling ODs, CODs, or even QODs on real and complex variables. The noncommutivity of the quaternions, as well as the increase to four real dimensions in each quaternion variable $\left(\mathbf{a}=a_{1}+a_{2} \mathbf{i}+a_{3} \mathbf{j}+a_{4} \mathbf{k}\right)$, contributes to this difficulty.

Our definition of symmetric-paired designs and our treatment of amicable designs lead to several open questions. Necessary and sufficient conditions must be determined for when CODs $A$ and $B$ can be constructed so that $A^{H} B$ is symmetric. Similarly, it must be determined when QODs $A$ and $B$ can be constructed so that $A^{Q} B=B^{Q} A$ or $B A^{Q}=A B^{Q}$. We expect that an analysis of symmetric-paired and amicable designs will be useful in future constructions of QODs

Our preliminary simulations involving the application of QODs as quaternion orthogonal space-time-polarization block codes, particular those involving the QOD presented in Example 11, are promising. The simulations suggest that certain QODs will be capable of achieving the same performance as complex orthogonal space-time-block codes that require twice the number of transmit and receive antennas. It remains to determine which QODs are optimally suited for application in space-time-polarization block coding. This determination will require further simulations and the further development of the theory of QODs. For example, the maximum rate and minimum decoding delay of QODs must be determined. In our future work, we plan to both advance the theory of these novel designs and further investigate their application as space-time-polarization block codes.

\section{ACKNOWLEDGMENT}

The authors would like to thank the reviewers for their detailed suggestions that greatly improved the presentation of this paper. The authors also would like to acknowledge that A. R. Calderbank, S. Das, N. Al-Dharhir, and S. N. Diggavi independently and concurrently investigated ideas concerning the use of matrices over the quaternions in the context of space-time block coding [3]. In part, they built a $4 \times 4$ space-time block code over the complex domain by using a $2 \times 2$ matrix whose entries are quaternion variables and whose rows are orthogonal. In their $2 \times 2$ matrix of quaternion variables, one of the entries is a nonlinear combination of the other entries. Hence, it is distinct from our proposed linear QODs. However, we include this acknowledgement to show that other authors are simultaneously studying different sorts of orthogonal matrices over the quaternion domain.

\section{REFERENCES}

[1] S. M. Alamouti, "A simple transmit diversity technique for wireless communications," IEEE J. Sel. Areas Commun., vol. 16, no. 8, pp. 1451-1458, Oct. 1998.

[2] S. L. Altmann, Rotations, Quaternions, and Double Groups. Oxford, U.K.: Clarendon, 1986.

[3] A. R. Calderbank, S. Das, N. Al-Dhahir, and S. N. Diggavi, "Construction and analysis of a new quaternionic space-time code for 4 transmit antennas," Commun. Inf. Syst., vol. 5, no. 1, pp. 1-26, 2005.

[4] B. S. Collins, "Polarization-diversity antennas for compact base stations," Microw. J., vol. 43, no. 1, pp. 76-88, Jan. 2000.

[5] B. S. Collins, "The effect of imperfect antenna cross-polar performance on the diversity gain of a polarization-diversity system," Microw. J., vol. 43, no. 4, pp. 84-94, Apr. 2000. 
[6] C. B. Dietrich, K. Dietze, J. R. Nealy, and W. L. Stulzman, "Spatial polarization and pattern diversity for wireless handheld terminals," IEEE Trans. Antennas Propag., vol. 49, no. 9, pp. 1271-1281, Sep. 2001.

[7] K. Finlayson, J. Seberry, T. Wysocki, and T. Xia, "Orthogonal designs with quaternion elements," presented at the 8th Int. Symp. Commun. Theory Appl. (ISCTA), Ambleside, U.K., Jul. 17-22, 2005.

[8] G. Ganesan and P. Stoica, "Space-time diversity using orthogonal and amicable orthogonal designs," in Proc. IEEE Int. Conf. Acoustics, Speech, Signal Process. (ICASSP), 2000, vol. 5, pp. 2561-2564.

[9] G. Ganesan and P. Stoica, "Space-time block codes: A maximum SNR approach," IEEE Trans. Inf. Theory, vol. 47, no. 4, pp. 1650-1656, Apr. 2001.

[10] A. V. Geramita, J. M. Geramita, and J. S. Wallis, "Orthogonal designs," Linear Multilinear Algebra, vol. 3, pp. 281-306, 1976.

[11] A. V. Geramita and J. M. Geramita, "Complex orthogonal designs," $J$. Combinatorial Theory, vol. 3, pt. Ser A, pp. 211-225, 1978.

[12] O. M. Isaeva and V. A. Sarytchev, "Quaternion presentations polarization state," in Proc. 2nd IEEE Topical Symp. Combined Optical-Microwave Earth Atmosphere Sensing, Atlanta, GA, Apr. 3-6, 1995, pp. 195-196.

[13] X.-B. Liang, "Orthogonal designs with maximal rates," IEEE Trans. Inf. Theory, vol. 49, no. 10, pp. 2468-2503, Oct. 2003.

[14] R. U. Nabar, H. Bölcskei, V. Ereg, D. Gesbert, and A. J. Paulraj, "Performance of multiantenna signalling techniques in the presence of polarization diversity," IEEE Trans. Signal Process., vol. 50, no. 10, pp. 2553-2562, Oct. 2002.

[15] P. J. Robinson, "Amicable orthogonal designs," Bull. Austral. Math. Soc., vol. 14, no. 2, pp. 303-314, 1976.

[16] P. J. Robinson and J. Seberry, "On the structure and existence of some amicable orthogonal designs," J. Austral. Math. Soc. (Ser. A), vol. 25, pp. 118-128, 1978.

[17] J. Seberry, S. A. Spence, and T. Wysocki, "A construction technique for generalized complex orthogonal designs and applications to wireless communications," Linear Algebra Appl., vol. 405, pp. 163-176, 2005.

[18] V. Tarokh, H. Jafarkhani, and A. R. Calderbank, "Space-time block codes from orthogonal designs," IEEE Trans. Inf. Theory, vol. 45, no. 5, pp. 1456-1467, Jul. 1999.

[19] J. S. Wallis, "Constructions for amicable orthogonal designs," Bull. Austral. Math. Soc., vol. 12, pp. 179-182, 1975.

[20] W. Wolfe, "Amicable orthogonal designs-existence," Canad. J. Math., vol. 28, no. 5, pp. 1006-1020, 1976.

[21] C. Yuen, Y. L. Guan, and T. T. Tjhung, "Orthogonal space-time block code from amicable complex orthogonal design," in Proc. IEEE Int. Conf. Acoust., Speech, Signal Process. (ICASSP), 2004, vol. 4, pp. 469-472.

[22] Z. Zhao, S. Stapleton, and J. K. Cavers, "Analysis of polarization diversity scheme with channel codes," in Proc. IEEE Veh. Technol. Conf. (VTC), 1999, pp. 1337-1381.

[23] Z. Chen, G. Zhu, J. Shen, and Y. Liu, "Differential space-time block codes from amicable orthogonal designs," in Proc. IEEE Wireless Commun. Netw., Mar. 2003, vol. 2, pp. 768-772.

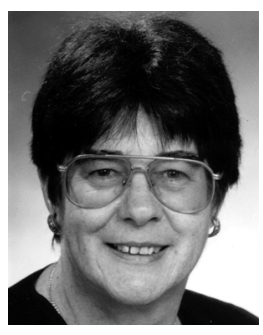

Jennifer Seberry (M'89-SM'97) received the Ph.D. degree in computation mathematics from La Trobe University, Melbourne, Australia, in 1971.

She has subsequently held positions at the Australian National University, the University of Sydney and the Australian Defense Force Academy (ADFA), The University of New South Wales. She has published extensively in discrete mathematics and is world renown for her new discoveries on Hadamard matrices and statistical designs. She started teaching in cryptology and computer security in 1980 . She is especially interested in authentication and privacy. In 1987, she founded the Centre for Computer and Communications Security Research at University College, ADFA, to be a reservoir of expertise for the Australian community. Her studies of the application of discrete mathematics and combinatorial computing via bent functions, S-box design, has led to the design of secure crypto-algorithms and strong hashing algorithms for secure and reliable information transfer in networks and telecommunications. Her studies of Hadamard matrices and orthogonal designs are applied in CDMA technologies. She is presently Professor of computer science at the University of Wollongong, New South Wales, Australia. She has over 300 publications and has successfully supervised $22 \mathrm{PhD}$ theses.

Dr. Seberry founded the AUSCRYPT/ASIACRYPT series of International Cryptologic Conferences in the Asia/Oceania area in 1990.

Ken Finlayson is a graduate student in the Centre for Computer and Information Security Research, School of Computer Science and Software Engineering, University of Wollongong, New South Wales, Australia.

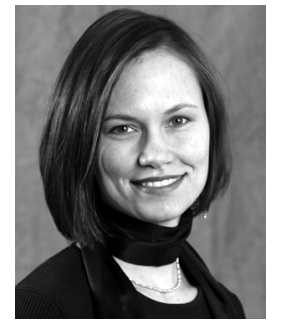

Sarah Spence Adams (M'05) received the B.S degree in mathematics (summa cum laude) from the University of Richmond, VA, and the M.S. and $\mathrm{Ph} . \mathrm{D}$. degrees in mathematics from Cornell University, Ithaca, NY, where she was also a member of the Wireless Intelligent Systems Laboratory in the Department of Electrical and Computer Engineering.

Previous experience includes appointments at the Institute for Defense Analyses, Center for Communications Research and the National Security Agency. She is currently an Assistant Professor at Franklin W. Olin College of Engineering, Needham, MA. Her research interests revolve around reliability in wireless communications.

Dr. Adams is a member of the American Mathematical Society, the Mathematical Association of America, and the Association for Women in Mathematics, and is an ExxonMobil Fellow in the Mathematical Association of America's Project NExT (New Experiences in Teaching).

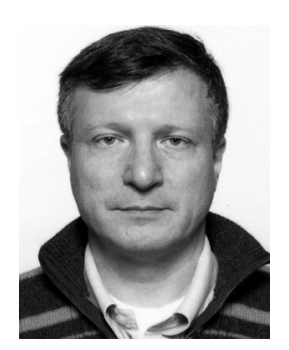

Tadeusz Antoni Wysocki (SM'98) received the M.Sc.Eng. degree with the highest distinction in telecommunications from the Academy of Technology and Agriculture, Bydgoszcz, Poland, in 1981 and the Ph.D. degree and the D.Sc. (Habilitation) degree in telecommunications from the Warsaw University of Technology, Warsaw, Poland, in 1984 and 1990 .

In 1992, he moved to a position at Edith Cowan University, Perth, Western Australia. In 1993, he was with the University of Hagen, Germany, within the framework of Alexander von Humboldt Research Fellowship. In December 1998, he became an Associate Professor, within the School of Electrical, Computer and Telecommunications Engineering, at the University of Wollongong, New South Wales, Australia. Since fall 2007, he has been with the University of Nebraska-Lincoln as Professor of Computer and Electronics Engineering at the Peter Kiewit Institute, Omaha, NE. The main areas of his research interests include space-time signal processing, diversity combining, indoor propagation of microwaves, as well as protocols for wireless ad hoc networks.

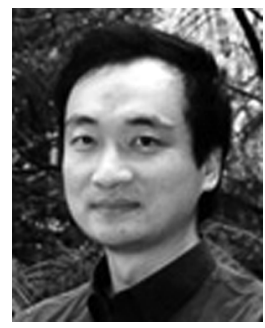

Tianbing Xia received the Ph.D. degree in computer security from the University of Wollongong, New South Wales, Australia, in 2001

He was a Software Engineer at the Department of Computer of China Construction Bank, Hubei branch, from 1995 to 1997 and Assistant Engineer from 1990 to 1994. In February 2001, he joined the faculty of informatics of the University of Wollongong as a Lecturer and was promoted to Senior Lecturer in 2005. His current research interests include combinatorial computing, Boolean function, orthogonal designs, mobile security, access control, computer security, and cryptography. His interest in other fields includes software engineering, client/server programming, and Web technology.

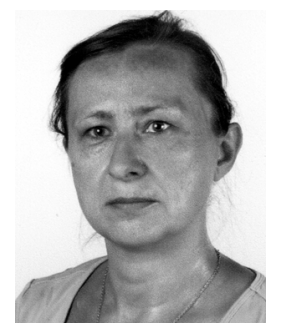

Beata Joanna Wysocki received the M.Eng. degree in electrical engineering from the Warsaw University of Technology, Warsaw, Poland, in 199 and the Ph.D. degree from the Australian Telecommunications Research Institute at Curtin University of Technology, Perth, Australia, in 2000. Her dissertation was on signal formats for code-division multiple-access wireless networks.

Since October 1999, she has been a Research Fellow with the Telecommunications \& Information Technology Research Institute at the University Wollongong, New South Wales, Australia. Her research interests include space-time signal processing, sequence design for direct sequence (DS) code-division multiple-access (CDMA) data networks, and optimization of ultra-wide-band (UWB) communication systems. 